\title{
Inverse Emulsion Polymerization for the Synthesis of High Molecular Weight Polyacrylamide and Its Application as Sand Stabilizer
}

\author{
Mahmoud A. Mohsin ${ }^{1}$ and Nuha F. Attia ${ }^{2}$ \\ ${ }^{1}$ Department of Chemistry, University of Sharjah, P.O. Box 27272, Sharjah, UAE \\ ${ }^{2}$ Abu Dhabi Polytechnic, P.O. Box 111499, Abu Dhabi, UAE \\ Correspondence should be addressed to Mahmoud A. Mohsin; mmohsin@sharjah.ac.ae
}

Received 13 April 2015; Revised 11 June 2015; Accepted 14 June 2015

Academic Editor: Gonzalo Martínez-Barrera

Copyright (C) 2015 M. A. Mohsin and N. F. Attia. This is an open access article distributed under the Creative Commons Attribution License, which permits unrestricted use, distribution, and reproduction in any medium, provided the original work is properly cited.

\begin{abstract}
Polyacrylamides constitute a class of polymers that can entirely dissolve or swell in water to form a solution or hydrogel, respectively. Free radical polymerization of acrylamide monomer, using both solution and inverse emulsion polymerization, was applied to produce polyacrylamide with various molecular weights. This investigation was focused on the production of polymers with varying molecular weight, depending on monomer to initiator ratio. Experimental conditions were designed to produce high molecular weight polymers that can be used in stabilization of sand dunes in the arid regions. Synthesized polyacrylamide samples were characterized using Gel Permeation Chromatography and solution viscosity in order to determine the molecular weights and molecular weights distribution. The rheological behavior was also investigated in different polymer concentrations and at various temperatures using Brookfield Rheometer. Lab-scale wind tunnel was used to determine the stability of the sand before and after treatment with the polymer. Compressive stress-strain test was also used to establish the mechanical behavior of the polymer-sand composite under controlled compressive load up to failure. The results showed that the use of high molecular weight polymer gave excellent mechanical and thermal stability.
\end{abstract}

\section{Introduction}

Arid Climate. The United Arab Emirates (UAE) climate is described as subtropical, warm, and arid. Air temperatures range from $35^{\circ} \mathrm{C}$ to $50^{\circ} \mathrm{C}$ around midday between May and October and from $20^{\circ} \mathrm{C}$ to $35^{\circ} \mathrm{C}$ during winter months. Deeper in the desert, the temperature on the ground during summer months can reach $70^{\circ} \mathrm{C}$ and can fall down to freezing in winter in some areas of the UAE. The average rainfall over the Emirates is less than $100 \mathrm{~mm}$ per annum. Moisture also condenses in the form of fog and dew especially on coastal belts. Strong winds and sand storms are of common occurrence throughout the Emirates and are frequent and severe especially during the summer. Sand dunes are a dominant landscape feature in the UAE.

Soils are generally coarse, sandy, and undeveloped. Most of the country is extremely arid, and sustained agriculture is not possible without artificial irrigation. For a successful agriculture, some protection against high velocity winds and moving sand is required. Sand dunes cover the area of north, west, and southwest of Al-Ain city in the eastern province of Abu Dhabi, UAE. These dunes vary from small crescentshaped to giant star and range in height from $10 \mathrm{~m}$ to $70 \mathrm{~m}$ above the ground level $[1,2]$. The accumulated sand after a storm has to be physically removed by using heavy earthmoving machinery in particular along the highway systems, townships, and plantation farms. To minimize sand invasion, barriers such as cement asbestos sheets, galvanized iron sheets, or date-palm fronts are erected across the prevailing wind direction to restrain further sand invasion and piles against these barriers are periodically removed [3]. Sand invasion and wind erosion reduce soil productivity causing loss of plant nutrients, degradation of soil structure, loss of storage capacity for water for plants, and a reduction in soil uniformity. Dust arises after a sand storm abrades and buries plants and blocks sunlight. In addition, clog streams and 
drainage channels damage water distribution systems and pollute drinking water with agricultural chemicals.

The inception of research of using chemicals as sand stabilizers dates back to the mid-1930s. More than half a century of research and practice has shown that chemical stabilizers are particularly suitable for the control of shifting sand and significantly help in reducing damage to vegetation and highway systems in deserts and arid zones, which are characterized by mobile or moving sand $[4,5]$. Chemical stabilization of sand forms a binding surface crust that conserves soil water beneath the crust that prevents or impedes wind erosion and stabilizes the sand. Depending on their chemical properties, sand stabilizers can form three types of binding crust: (i) a rigid crust, (ii) a flexible crust, or (iii) an elastic crust. All these crusts have smooth surfaces that protect the sand surface from direct erosion by strong winds [5,6]. Ideal chemical sand stabilizers should offer good adhesion and rapid infiltration of the sand when a liquid stabilizer is sprayed onto the sand surface.

As early as 1934, scientists started to study the stabilization of shifting sands using asphalt emulsions. In 1959, scientists conducted dune stabilization experiments using synthesized polymer solution to replace the asphalt. Following this, in 1964, scientists performed a series of dune stabilization experiments in the laboratory as well as in the field. They obtained a large amount of data on their infiltration rates and depths. Between 1969 and 1972, researchers conducted shifting-sand stabilization experiments using a chemical called Nerosine, and in recent years, they have tested butadiene-styrene latex [4]. Other scientists have developed sand stabilizers such as urea-formaldehyde, urea-dicyandiamide, and polyacrylamide [7]. Researchers have also tested more than 30 kinds of organic and inorganic materials to control wind erosion. Other countries, including Germany, Iran, Libya, Algeria, Saudi Arabia, China, Egypt, United Arab Emirates, and Iraq, have also conducted dune stabilization experiments using oil products. These systems were used to stabilize sandy areas disturbed by wind erosion or construction activities, to protect roads, buildings, and valued farmed areas from encroachment by blowing sand [8-12]. In areas that are subject to strong winds, chemical stabilizers keep soil and sand from blowing off the ground and potentially being deposited in nearby wetlands, watercourses, roads, and sewers. Chemicals, which are used as sand stabilizers, including both organic and inorganic substances, must be nontoxic, nonpolluting, adaptable to the climatic and environmental conditions in the area being treated, highly effective, and long lasting. In the UAE, a number of field trials have been carried out in order to combat encroachment and to halt sand dune movements. Various methods were used, such as the erection of fences and barriers, protective forestation, and leveling followed by covering and spraying with crude oil or other oil stabilizers.

Petroleum mulch was also used for dune stabilization, and it seemed to have given better results; however, the area sprayed produced dirty appearance and possibly polluted the local environment. It would be worthwhile to try other sand stabilizers, which do not produce the bad effects of petroleum mulch. Another method, which can be regarded as more environmentally friendly, was planting the arid zone with species such as Acacia and Calligonum, but this proved to be very expensive to maintain over a long period of time. In this work, a high molecular weight sand stabilizer based on polyacrylamide was synthesized by both solution and inverse emulsion processes to produce polymers in powder and emulsion forms, respectively, for easy application. These materials were applied for wind erosion control and sand dune stabilization in Al-Ain town of the UAE.

Inverse Emulsion Polymerization. Acrylamide based polymers or copolymers of high molecular weight can be obtained by solution polymerization in aqueous media. However, solution polymerization is limited to relatively low monomer concentrations because of the high viscosity of the final polymer or copolymer solution. These difficulties are overcome by using heterophase water-in-oil (inverse emulsion) polymerizations. This process involves the dispersion of an aqueous monomer(s) solution in an aliphatic continuous oil phase. The polymerization takes place in a batch reactor under inert atmosphere using free radical initiator. Temperatures ranging between 25 and $55^{\circ} \mathrm{C}$ are employed with continuous vigorous agitation. There has been a considerable progress in the field of inverse emulsion polymerization since the pioneering work of Vanderhoff [13].

This study focuses on the examination of the molecular weight distribution of a water-soluble polyacrylamide, which was polymerized in solution and in inverse emulsion (water in oil) polymerization. The shape of this distribution as a function of the amount of chain transfer agent (Sodium formate) and of initiator was examined to obtain information on the mechanisms involving free radical polymerization.

In a water-in-oil polymerization, a hydrophilic monomer (usually in aqueous solution) was dispersed in a continuous organic phase using a water-in-oil emulsifier. The free radical polymerization was carried out to yield inverse latex, that is, a colloidal dispersion of water swollen polymer particles in oil. Such a process is well suited to the preparation of high molar mass polymers at rapid reaction rates $[14,15]$. It should be mentioned at this stage that the economic advantages of using the synthesized polyacrylamide in this work compared to the commercial ones are perhaps not that significant. However, it could be very much economical if a commercial plant would be built locally to produce such polymer on a large scale. The important issue is that the commercial polymers, when applied in the UAE, will degrade the polymer significantly due to the extreme weather conditions. Full degradation study of the synthesized polymer will be explored in future research.

\section{Experimental}

2.1. Materials. Acrylamide (AM) monomer was recrystallized twice from chloroform. The purity of transfer agent (sodium formate) (A) was measured and an aqueous solution (5 wt $\%$ ) of transfer agent was added to the reaction solution for selected experiment. Deionized water was used in all experiments. Potassium persulfate (KPS) was used as the initiator (I), purified by recrystallization with methanol, dried 
in a vacuum, and stored at $5^{\circ} \mathrm{C}$ in a freezer. For inverse emulsion polymerization, the oil was a narrow cut isoparaffinic mixture and was used as supplied. The emulsifier was a polymeric surfactant, which also contained some sorbitan monooleate. Sodium ethylene diamine tetraacetic acid was used as a chelating agent, to eliminate catalysis of persulfate decomposition by traces of transition metal ions. All chemicals were purchased from Sigma-Aldrich Chemicals Company.

2.2. Polymer Synthesis. Solution polymerizations were carried out in a $5 \mathrm{~L}$ jacketed glass reactor fitted with a mechanical stirrer, thermocouple, and a condenser. The reactor temperature was controlled by using a circulating water bath. The reactor was also connected to a Nitrogen gas inlet. The starting recipe (without the KPS solution) was first purged with purified Nitrogen to remove most residual Oxygen. The reaction temperature was maintained between $50^{\circ} \mathrm{C}$ and $60^{\circ} \mathrm{C}$. The initiator solution was then injected after thermal equilibrium.

Emulsion polymerizations were performed using the same reactor settings. The aqueous phase and oil phase were prepared separately. The aqueous phase was then poured into the reactor, which contained the oil phase, under vigorous stirring. The emulsion was formed and continuously purged with purified Nitrogen gas. The agitation rate was fixed first at $200-350 \mathrm{rpm}$ and then increased to $3000 \mathrm{rpm}$ when all the ingredients were added and the reaction temperature achieved the desired setting of $50^{\circ} \mathrm{C}$. Initiator solution with a specific concentration was then injected into the reactor. For each procedure (solution and emulsion polymerization), three experiments were carried out as follows: (a) reference experiment, (b) where the concentration of initiator was doubled (" $2 \mathrm{I}$ "), and (c) where the concentration of the transfer agent was divided by three (“A/3").

Solution polymerizations were stopped after 20 minutes, and the polymer was analyzed. However, the emulsion polymerization reactions were kept continuously going, and two aliquots were withdrawn at 20-minute intervals for analysis. Following this, the reaction was then terminated.

2.3. Polymer Characterization. Polymer characterization was conducted simultaneously in order to achieve a polymer with high molecular weight. Characterization techniques including NMR and FTIR were used for routine analysis. Gel Permeation Chromatography (GPC) and solution viscosity were also used continuously for the determination of molecular weight and molecular weight distribution. For both, solution and emulsion polymerizations, polyacrylamide was recovered from the reaction medium by precipitation in excess methanol containing a small amount of hydroquinone, followed by several washings and drying overnight in an oven at $45^{\circ} \mathrm{C}$. GPC analysis was carried out with an Agilent HPLC/GPC (1100 Series) with ultraviolate (UV) and Refractive Index (RI) detectors. The mobile phase consisted as a solution containing sodium nitrate $(0.25 \mathrm{M})$ and sodium hydrogen phosphate $(0.01 \mathrm{M})$. Two mix bed Agilent hydrogel columns were used. Calibration was carried out with narrow molecular weight distribution water-soluble polyethylene oxide (PEO) standards (twelve standards covering a wide range of molecular weights). Solution viscosity measurements for the determination of viscosity molecular weight $M_{v}$ were also used to verify the molecular weight values.

2.4. Polymer Rheology. The application of polyacrylamide as a sand dune stabilizer ultimately depends on the way in which it is sprayed on the field or on the sand dune. Rheological properties and how the polymer solution behaves at various concentrations and temperatures were examined. Factors affecting these properties were also determined. The Brookfield Viscometer model LVDV- II+ was used to determine the shear viscosity of the polymer sample. This instrument applies Hooke's law which states that the force $(F)$ required to extend or compress a spring by some distance $(x)$ is directly proportional to that distance. That is, $F=K x$, where $K$ is a constant factor characteristic of the spring and its stiffness. This equation was used to calculate the torque produced between the liquid in the concentric cylinder. The measurement of viscosity and the behavior of the viscous flow at different concentrations and temperatures were examined. The Brookfield Viscometer model LVDV- II+ with small sample adapter has a cylindrical spindle, which rotates in the fluid to measure the torque needed to overcome the viscous resistance to the inducted movement. The spindle is inserted in the test material until the fluid level reaches the immersion mark on the spindle's shaft at a constant temperature. The motor speed was turned on, and the readings of viscosity in centipoise $(\mathrm{cP})$ and shear stress versus different shear rates were taken. To start the experimental part, the viscometer was calibrated and the viscosity was measured for a series of prepared polymers with different concentrations for all polymers which had been prepared by both emulsion and solution polymerization. At a constant temperature, the polymer solution was poured into the viscometer cell to determine the values of the shear stress, the shear rate, and the viscosity. A series of graphs were obtained for varying concentrations of different polymers at a constant temperature.

2.5. Wind Tunnel Experiment. Laboratory scale wind tunnel (Armfield Technical Education Company Limited, Ringwood, Hampshire, England) was used to simulate the wind pattern for the wind erosion experiment. The wind speed was calibrated and monitored by a manometer.

For laboratory experiments, rectangular shape plastic trays with fixed dimension (length $12 \mathrm{~cm} \times$ width $4 \mathrm{~cm} \times$ height $2 \mathrm{~cm}$ ) were filled with known weight of local sand and were placed on the tunnel floor upwind. The effect of flow interference from the surrounding trays was ignored. Sand erosion tests were conducted under three different wind speed levels $\left(4,5\right.$, and $\left.10 \mathrm{~m} \cdot \mathrm{s}^{-1}\right)$ measured by a nanometer on the side of the tunnel. During the test, three trays containing sand, which has been treated with the sand stabilizer, were placed next to each other. The fourth tray was placed $10 \mathrm{~cm}$ away from the side of the other three. This tray was filled with a known weight of the same sand but without any stabilizer treatment. This will be used as a control sample for comparison. All four trays were placed at the flat surface of the wind tunnel and facing the upwind direction. 
TABLE 1: Recipes of solution (S) and emulsion (E) polymerization experiment of acrylamide at $50^{\circ} \mathrm{C}$. All quantities are given in grams. The weight average molecular weight $M_{w}$ was calculated from the GPC and the viscosity average molecular weight $M_{v}$ was calculated from viscosity measurement and given in $\mathrm{g} \cdot \mathrm{mol}^{-1}$.

\begin{tabular}{|c|c|c|c|c|c|c|c|c|c|c|}
\hline Sample number & Sample code & Polymer & Monomer Am & Water & Initiator & Surfactant & Oil & Transfer agent $\mathrm{A}$ & Mol. wt. $M_{w}$ & Mol. wt. $M_{v}$ \\
\hline 1 & $S$ & PAM-Sa & 52.4 & 3000 & 0.1 & 0 & 0 & 53.7 & $5.0 \times 10^{5}$ & $4.1 \times 10^{5}$ \\
\hline 2 & S:2I & PAM-Sb & 52.4 & 3000 & 0.2 & 0 & 0 & 53.7 & $2.4 \times 10^{5}$ & $1.9 \times 10^{5}$ \\
\hline 3 & $\mathrm{~S}: \mathrm{A} / 3$ & PAM-Sc & 52.4 & 3000 & 0.1 & 0 & 0 & 17.9 & $7.5 \times 10^{5}$ & $6.6 \times 10^{5}$ \\
\hline 4 & $\mathrm{E}$ & PAM-Ea & 52.4 & 900 & 0.1 & 212 & 2100 & 53.7 & $1.4 \times 10^{6}$ & $7.0 \times 10^{5}$ \\
\hline 5 & $\mathrm{E}: 2 \mathrm{I}$ & PAM-Eb & 52.4 & 900 & 0.2 & 212 & 2100 & 53.7 & $8.3 \times 10^{5}$ & $5.1 \times 10^{5}$ \\
\hline 6 & $\mathrm{E}: \mathrm{A} / 3$ & PAM-Ec & 52.4 & 900 & 0.1 & 212 & 2100 & 17.9 & $2.2 \times 10^{6}$ & $1.8 \times 10^{5}$ \\
\hline
\end{tabular}

2.6. Mechanical Properties. Compression test was used to determine the mechanical behavior of the polymer-sand composite under escalating compressive load until failure is reached. Compressive stress and strain were calculated and plotted as stress-strain diagram was used to measure the load bearing capacity in compression and the stiffness (modulus of elasticity) for the polymer composites. Other parameters such as the peak load, peak stress, and elongation at peak were also determined. Composite specimens were prepared by mixing various weight percentages of the highest molecular weight polymer $\left(2.2 \times 10^{6} \mathrm{~g} / \mathrm{mol}\right)$ obtained from the inverse emulsion process with $300 \mathrm{~g}$ of sand and compressed in a cylindrical mold. The mold was then placed in an oven held at $50^{\circ} \mathrm{C}$ for 48 hours to completely evaporate the excess water which was used to dissolve the polymer. The detailed sample compositions and mixing components are given in Table 1. At least four samples for each composition were made for the compressive test. Compressive strength for all six fully dried composite samples with varying weight percentage of the polymer was determined using $100 \mathrm{KN}$, MTS universal testing machine under displacement controlled conditions.

The tests were conducted at ambient temperature and under a constant cross head speed (CS) of $0.05 \mathrm{~cm} / \mathrm{min}$ until the test sample fractured. At least three valid results for each sample have been completed in order to calculate the mechanical properties. The average value of the results obtained from three valid samples was calculated and presented herein. Cylindrical shaped test composite (diameter of $50 \mathrm{~mm}$ and thickness of $50 \mathrm{~mm}$ ) was loaded across its diameter and subjected to static compression test with escalating compressive load until sample failure occurred. Such loading generates a tensile stress at the center of the cylinder in a direction perpendicular to the direction of applied load. Tensile strengths for each specimen $(\sigma)$ were calculated using the following equation:

$$
\sigma=\frac{2 P}{\pi t D}
$$

where $P=$ maximum applied compressive load recorded during the test (Newton), $D=$ diameter of the test specimen $(\mathrm{mm})$, and $t=$ thickness of the test specimen $(\mathrm{mm})$.

\section{Results and Discussions}

As for the synthesis and characterization of all polymers that were prepared using both solution (S) and emulsion (E) polymerization methods, three polymers were synthesized in each method and were characterized by NMR and FTIR. All polymers showed typical bands associated with stretching and bending of various groups and in particular the carbonyl and the amine groups. Details of characterization were presented elsewhere $[16,17]$. Table 2 shows the specific experimental conditions for each batch of polymerization in solution and emulsion method. Those synthesized polymers by solution polymerization were given codes: PAM-Sa, PAM$\mathrm{Sb}$, and PAM-Sc, referring to the method of synthesis and condition (PAM is referring to polyacrylamide); the capital letter $S$ refers to solution polymerization and the letter (a) is for the reference or standard experiment; (b) is for doubling the initiator concentration (2I), while the letter (c) is for the reduced concentration of the transfer agent by one-third $(\mathrm{A} / 3)$. Polymer obtained using emulsion polymerization followed the same coding with the letter $\mathrm{E}$ to refer to emulsion method.

Molecular weight measurements were obtained from two different techniques, namely, Gel Permeation Chromatography (GPC) and dilute solution viscosity. Synthesized polymers were characterized by their intrinsic viscosity. The viscosity average molecular weight of the polymers was estimated from the intrinsic viscosity data at an appropriate temperature $\left(30^{\circ} \mathrm{C}\right)$ in a specific solvent (distilled water). All viscosity measurements were performed using an Ubbelohde type viscometer immersed water bath held at $30^{\circ} \mathrm{C}$.

The data was calculated and listed in Table 1. A reasonable agreement was observed between the two molecular weight values obtained from the two different techniques. This could primarily be due to the use of ethylene oxide standard for the calibration of the GPC system. The value of molecular weight obtained using emulsion polymerization was much higher than those obtained using solution polymerization. It is possible to increase the molecular weight of the polymer to even a higher extent if the viscosity of the reaction solution is reduced and the monomer/initiator ratio is increased. Emulsion polymerization has some advantages over the solution method in which the resulting polymer can readily be used as sand stabilized after making the required concentration. In addition, the control of the reaction exotherm is much easier, and the solution viscosity can be managed better to ensure the maximum mixing of reacting materials.

Figure 1 shows the molecular weight distribution obtained from the GPC system for the three polymer samples, which 
TABLE 2: Composition of six polymer-sand composite samples with different weight $\%$ of the polymer.

\begin{tabular}{|c|c|c|c|c|c|c|}
\hline Sample number & 1 & 2 & 3 & 4 & 5 & 6 \\
\hline Solid PAM g & 0.33 & 0.66 & 0.99 & 1.33 & 1.66 & 1.99 \\
\hline Mix of PAM solution with $300 \mathrm{~g}$ of sand & $13 \mathrm{~mL}$ & $26 \mathrm{~mL}$ & $39 \mathrm{~mL}$ & $52 \mathrm{~mL}$ & $65 \mathrm{~mL}$ & $78 \mathrm{~mL}$ \\
\hline Constant $\mathrm{H}_{2} \mathrm{O} \%$ & 2.5 & 2.5 & 2.5 & 2.5 & 2.5 & 2.5 \\
\hline Weight $\%$ of PAM in $300 \mathrm{~g}$ of sand & 0.11 & 0.22 & 0.33 & 0.44 & 0.55 & 0.66 \\
\hline
\end{tabular}

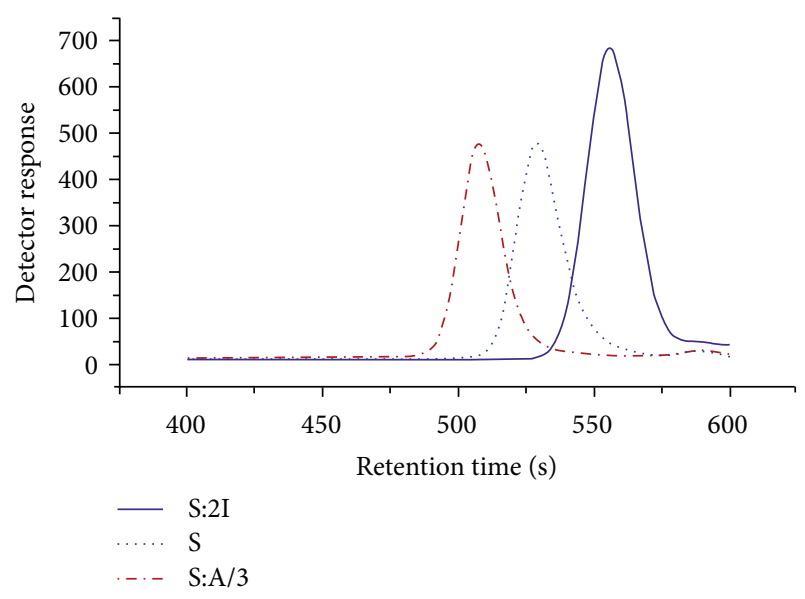

FIGURE 1: Molecular weight distribution curves for three polymers obtained in solution polymerization.

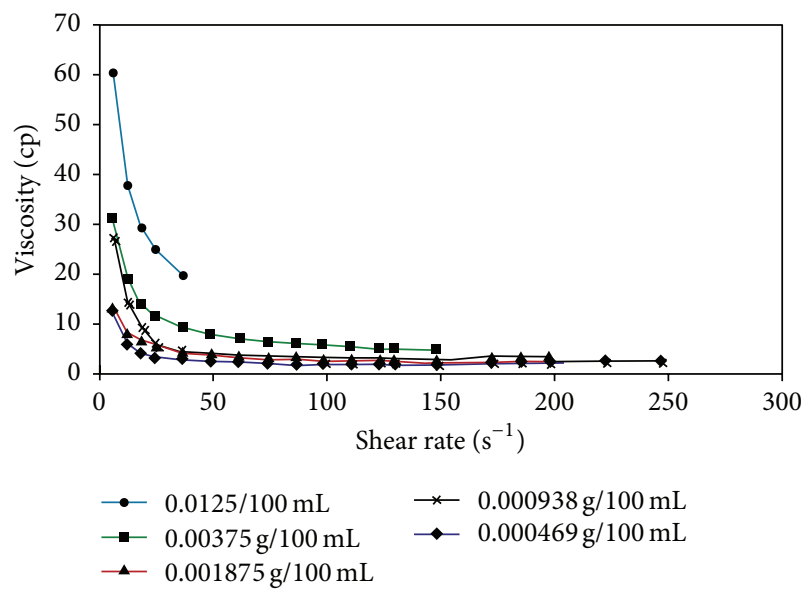

FIGURE 2: Shear rate versus viscosity for PAM-Sa at $20^{\circ} \mathrm{C}$ at different concentrations.

were polymerized in solution method. The polydispersity indexes for these polymers were excellent as they were between 1.05 and 1.10, which is not usually achieved in a free radical polymerization.

Figure 2 shows the shear rate versus solution's viscosity at $20^{\circ} \mathrm{C}$ for different polymer concentrations. As one can see from the graph, the concentration of the polymer solution contributed to the increase in viscosity. In addition, the increase of molecular weight increased the viscosity of the polymer at constant concentration and temperature. As the shear rate increased, the viscosity decreased because it breaks down the polymer chains resulting in a less viscous solution. The viscosity can be said to be non-Newtonian and shear thinning for high concentrations, while at low concentrations the viscosity behavior is almost the same with a Newtonian behavior. Lower viscosity is desirable in the current application in which the polymer solution has to be spread over the sand dune in order to form a thin film to stabilize the sand.

Before conducting tests on the sand, $0.1 \%$ polymer stock solutions (based on total solids) were prepared. In this study, deionized water produced by a Millipore laboratory water purification system was used. The polymer was dissolved in warm water $\left(25^{\circ} \mathrm{C}\right.$ to $\left.35^{\circ} \mathrm{C}\right)$; then stirred for 30 minutes to form $0.1 \%$ aqueous solutions. This concentration was adopted for all experiments in order to control the solution viscosity by keeping it to a minimum, allowing the spraying process to be easy and uniform. Two types of sand structures and formations were constructed outside the laboratory using locally available sand. One of the structures was a flat surface and the other was a dune or hill profile. Both formations were sprayed with the polymer solution prepared earlier and left to the effect of local wind and temperatures. These structures were visually inspected over a period of time. Photographs of the sand dune patterns were taken at regular intervals to study the effects of wind on these formations. Figure 3 shows the dune pattern over the entire period after the application of the sand stabilizer. Figure 3(a) was taken during the first week of application; Figure 3(b) was taken after three weeks; and Figure 3(c) was taken after nine weeks of exposure to local weather.

The dune was divided into two halves using a marking tape; the left hand side of the dune was treated with the polymer solution and the other half was used as a control. It was clear from Figures 3(a), 3(b), and 3(c) that the prepared polymer worked reasonably well in holding the sand from drifting under the effects of the local wind. It should be noted that the yearly average wind speed in Al-Ain city is 5 meters per second or just above 18.5 kilometer per hour [1]. This is considered to be light to moderate all year around. Such wind force is capable of moving and depositing large volumes of sand on local highways, vegetation farms, and residential areas. Application of sand stabilizers could potentially solve these problems by preventing accumulation of sand in these crucial areas.

On the flat surface, the polymer's performance was as good as on the dune pattern. Two wooden trays of equal dimensions were filled with local sand. As in the previous experiment, the right hand tray, this time, was treated with the polymer solution, and the left hand side was left without 


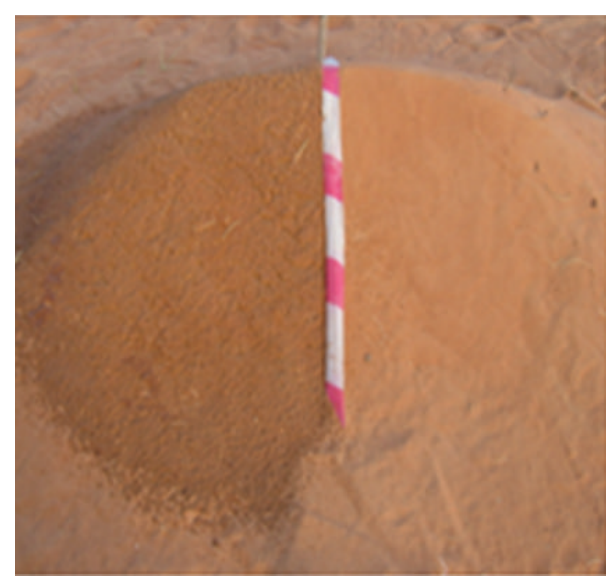

(a)

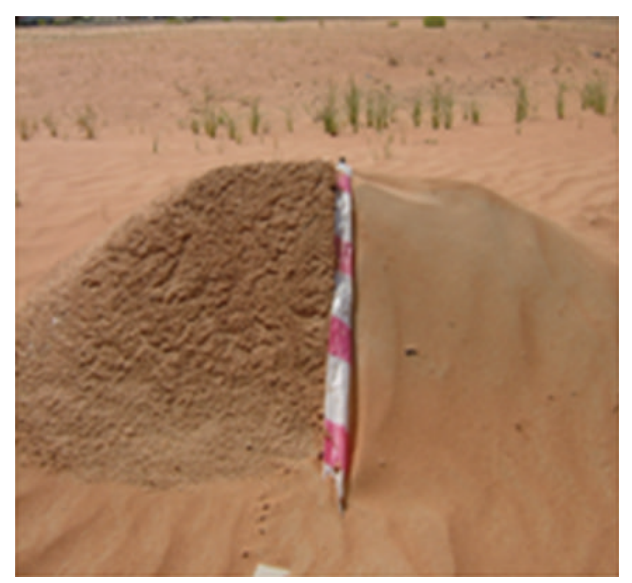

(b)

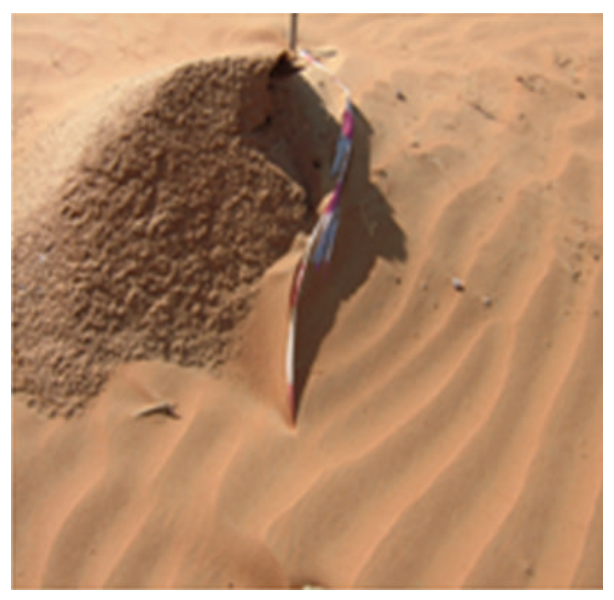

(c)

Figure 3: Shows sand erosion in a dune formation over 9-week exposure to weathering condition in Al-Ain in which the treated half is completely preserved. (a) After one week, (b) after three weeks, and (c) after nine weeks.

treatment. These trays were placed on a flat surface in an open air and fixed to the ground. Figure 4 shows the sand erosion over a period of nine weeks. After nine weeks of exposure to the local weather and wind, the sand in the left hand tray had completely been dispersed and was removed out of the tray by the effect of wind. On the other hand, the sand, which had been treated with the prepared sand stabilizer, remained unchanged over the same period of time. This effect can be shown in Figures 4(a), 4(b), and 4(c).

For more accurate assessment of the durability of the sand stabilizer and its stabilizing strength over the sand, a laboratory-scale wind tunnel was used to carry out a number of experiments at different wind speeds, namely 4, 5, and $10 \mathrm{~m} \cdot \mathrm{s}^{-1}$, bearing in mind that the annual average wind velocity in Al-Ain city is $5 \mathrm{~m} \cdot \mathrm{s}^{-1}$. In this test, the sand was collected from nearby dune sites and used for the evaluation. The sand was used to fill four identical rectangular trays. Three of these trays were sprayed with the polymer solution and left over night at room temperature to dry. Only one tray was left without the polymer and was used as a control. Those four trays were placed inside the wind tunnel and the wind speed was selected. To determine the correct weight loss, the trays containing the sand sample were weighed before and after the test using an electronic balance with an accuracy of $0.1 \mathrm{~g}$. The weight loss at the end of the test corresponded to the sand blowing off at particular wind speeds. The weight loss was monitored with time and recorded every 5 minutes. Figure 5 shows the sand weight loss versus the time at $5 \mathrm{~m} \cdot \mathrm{s}^{-1}$ wind speed. The weight loss for the three trays filled with sand, which were sprayed with the polymer, was found to be zero as the final weight remained the same as the original weight. This is clear evidence that the polymer stabilizer has held the sand grains together and prevented them from blowing off with the wind. On the other hand, the weight loss from the control sample exhibits a gradual weight loss with time. In fact, after 30 minutes of exposure, the tray has lost almost $50 \%$ of its original weight. At this stage, the wind direction was changed by $180^{\circ}$; and a further weight loss of $25 \%$ has occurred. Finally, only less than $25 \%$ of the original sand weight was left in the tray after 45 minutes of inside exposure to the wind tunnel. The latter happened as the sand has accumulated and sheltered around the edges of the tray. 


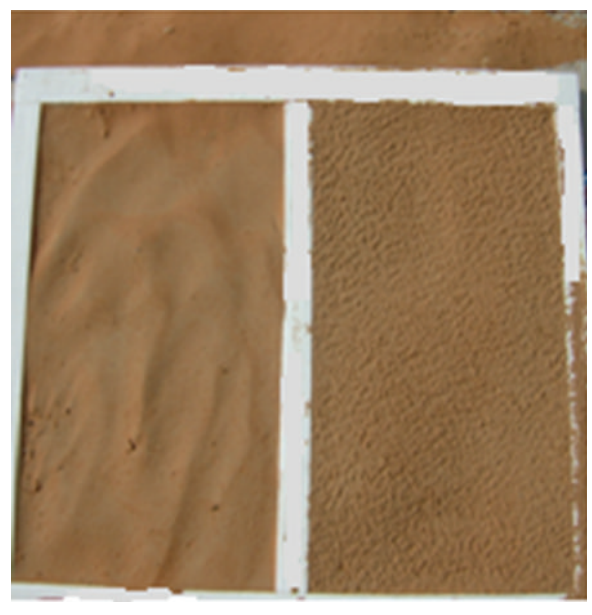

(a)

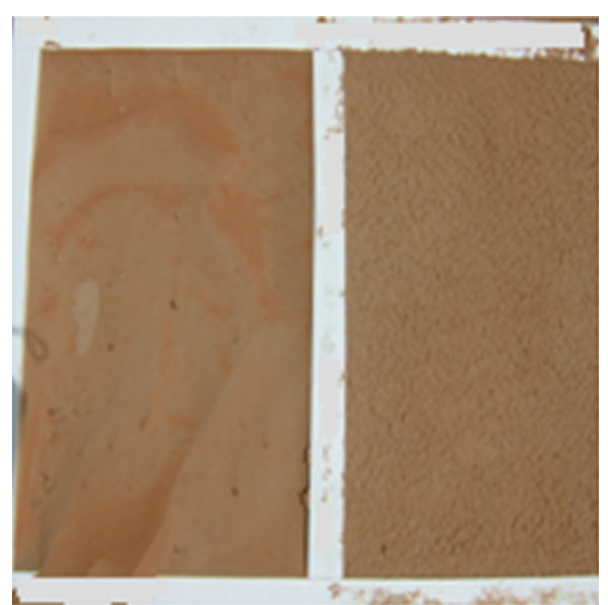

(b)

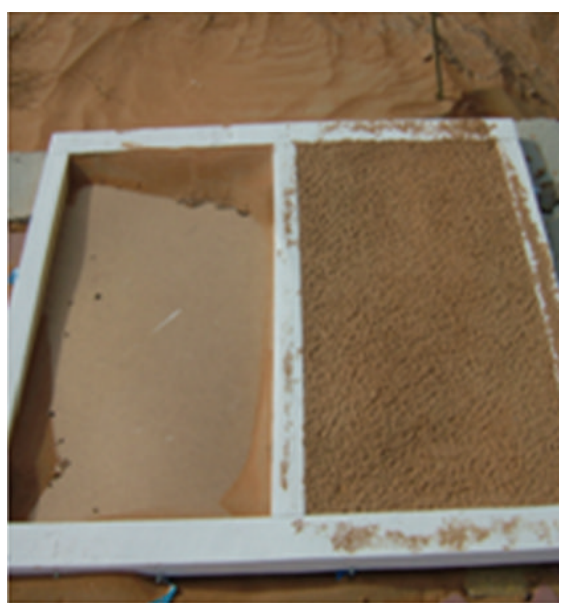

(c)

FIgURE 4: Shows sand erosion in a flatbed formation over 9-week exposure to weathering condition in Al-Ain in which the treated half is completely preserved. (a) After one week, (b) after three weeks, and (c) after nine weeks.

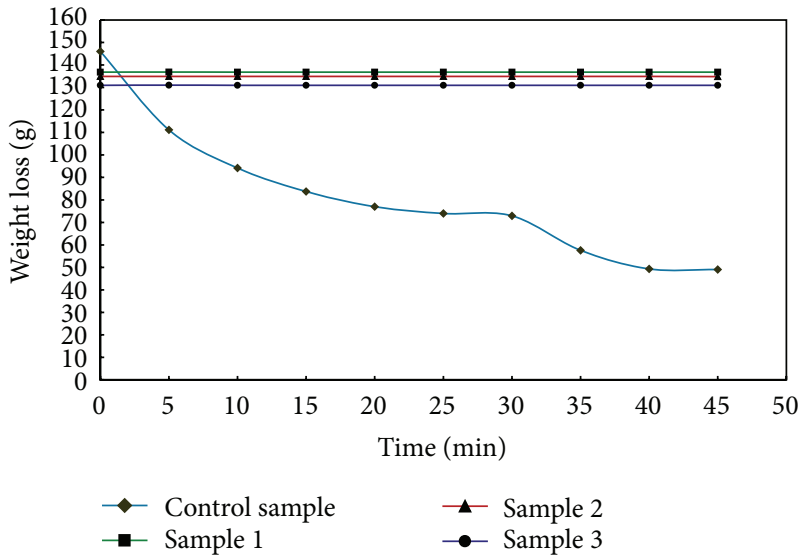

Figure 5: Weight loss versus time at $5 \mathrm{~m} / \mathrm{s}$ wind speed.

When the wind speed was lowered to $4 \mathrm{~m} \cdot \mathrm{s}^{-1}$, a similar trend was observed, but with a reduced weight loss from the control tray. Only $11 \%$ of the total sand weight was lost as depicted in Figure 6.

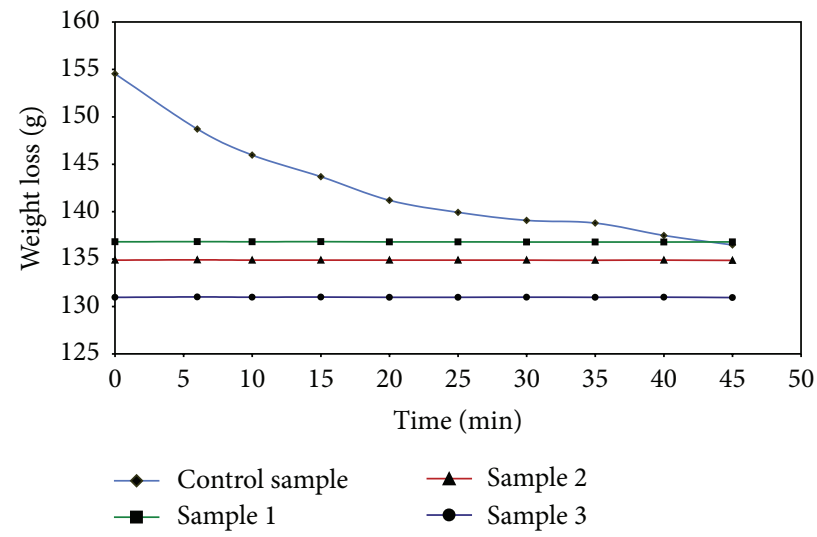

FIGURE 6: Weight loss versus time at $4 \mathrm{~m} / \mathrm{s}$ wind speed.

Once again, the treated trays remained unaffected by the reduced wind speed. Figure 7 illustrates the sand weight loss versus time at $10 \mathrm{~m} \cdot \mathrm{s}^{-1}$ wind speed. Only 4 minutes were required to reduce the sand weight by almost $75 \%$. The wind 


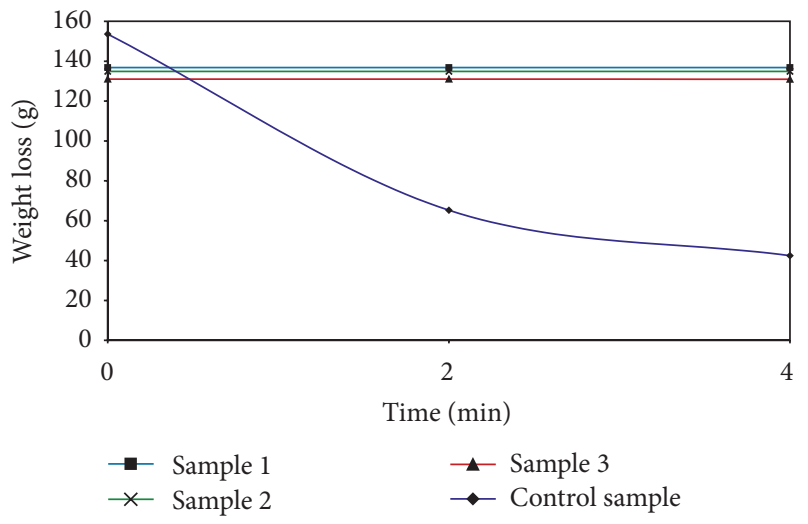

FIGURE 7: Weight loss versus time at $10 \mathrm{~m} / \mathrm{s}$ wind speed.

at this speed is considered as fresh breeze, and yet it can blow off almost $75 \%$ of the sand within very short time. Wind blowing is a major agent in the weathering process, and wind erosion is common in the Gulf region where soils are generally unprotected and prevailing winds transport the exposed surface materials.

This movement of sand and soil, referred to as aeolian transport, is common throughout the United Arab Emirates. In addition, wind direction during sand storms often fluctuates, allowing the movement of sand which was previously deposited by wind from a slightly different direction. Blow-sand damage to both urban and agricultural uses can result in substantial economic losses. Damage to agriculture during a sand storm can result in deposition from an adjacent area or on-site erosion of the topsoil. Urban related damage is generally limited to equipment, buildings, and highways. It is, therefore, necessary to conduct large-scale or field experiment to verify the finding of these research results.

Figure 8 demonstrated a typical stress-strain diagram obtained from the MTS universal testing machine.

The average data for three successful test samples obtained from the MTS machine are given in Table 3.

The modulus and the tensile strength of the polymer-sand composite were directly obtained from the MTS machine for various polymer weight percentages. These mechanical properties are predominant for all composite materials and can be used to characterize them for various applications. Figure 9 shows the effect of increasing polymer weight \% on the modulus of the prepared sample.

When polymer weight percent was $0.33 \%$, the modulus obtained was very low reaching less than $10 \mathrm{MPa}$. When the polymer weight $\%$ in the composite was doubled, the Modulus increased by more than 15 -fold reaching a value of $85 \mathrm{MPa}$. The substantial increase in modulus can be attributed to the binding strength of the polymeric material and also to the polymer having very high molecular weight represented by very long chain length where chain entanglement constitutes the prevailing forces. The trend in modulus was abrupt when the polymer weight percentage increased from

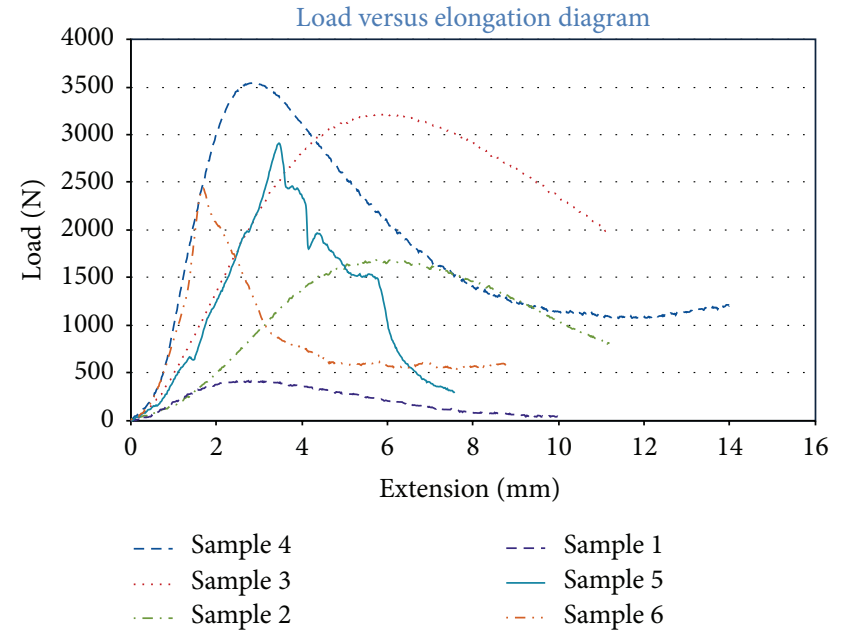

FIGURE 8: Typical load-deformation diagram obtained from the MTS universal testing machine for six test specimens with different polymer's weight percentage.

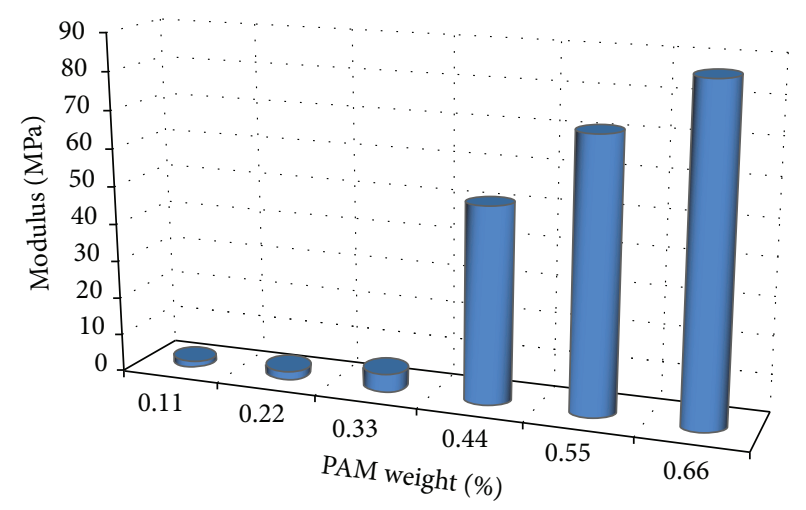

Figure 9: Modulus of elasticity of sand-polymer composites containing various polymer weight percent.

$0.33 \%$ to $0.66 \%$ while the increase in the tensile strength was gradual as shown in Figure 10. It was also observed that the calculated value of the tensile strength was $0.6 \mathrm{MPa}$ at $0.33 \%$ polymer weight and increased to $0.9 \mathrm{MPa}$ when the polymer percentage was doubled.

\section{Conclusions}

Reverse-emulsion polymerization (water-in-oil) of acrylamide monomer was conducted under three different experimental conditions to produce polyacrylamide with high molecular weight. In contrast, solution polymerization was also used to prepare polyacrylamide, using three different experimental conditions. Characterizations of these polymers were performed using NMR, FT-IR, GPC, and Rheology and were typical for polyacrylamide. The aim of this research was to synthesize polyacrylamides using two different methods and exploiting their physical properties in binding the surface particles together with the soil or sand to 
TABLE 3: Mechanical properties data obtained from the MTS universal testing machine (average of three samples).

\begin{tabular}{lcccccc}
\hline $\begin{array}{l}\text { Specimen } \\
\text { number }\end{array}$ & $\begin{array}{c}\text { Peak load } \\
\text { (Newtons) } \mathrm{N}\end{array}$ & Peak stress MPa & Modulus MPa & $\begin{array}{c}\text { Cylindrical area } \\
\left(\mathrm{mm}^{2}\right)\end{array}$ & $\begin{array}{c}\text { Elongation at peak } \\
(\mathrm{mm})\end{array}$ & $\begin{array}{c}\text { Tensile strength } \\
(\sigma) \mathrm{MPa}\end{array}$ \\
\hline 1 & 417.81 & 0.20 & 1.574 & 7857 & 7.293 & 0.11 \\
2 & 1686.29 & 0.70 & 2.644 & 7857 & 2.875 & 0.43 \\
3 & 2429.96 & 0.95 & 5.044 & 7857 & 2.983 & 0.62 \\
4 & 2909.89 & 1.13 & 50.905 & 7857 & 2.868 & 0.74 \\
5 & 3216.95 & 1.28 & 70.956 & 7857 & 1.725 & 0.82 \\
6 & 3538.95 & 1.40 & 86.413 & 7857 & 0.90 & 1.710 \\
\hline
\end{tabular}

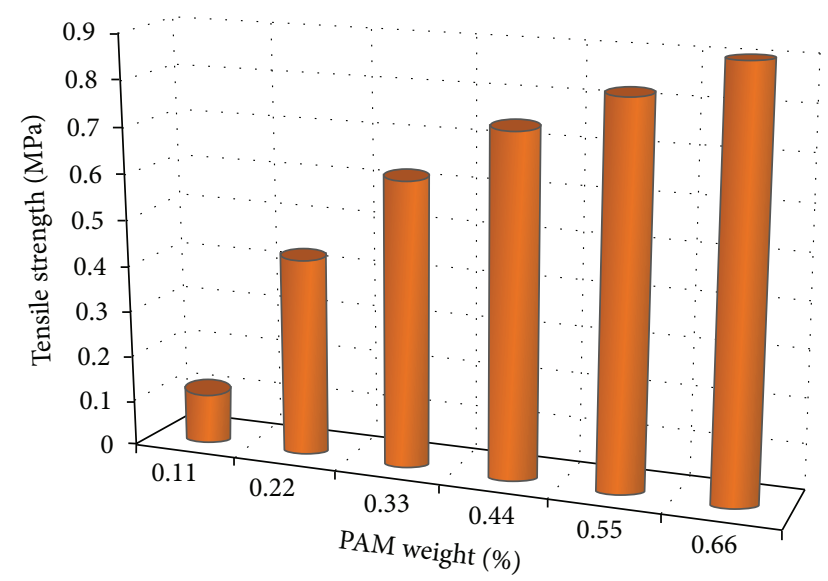

Figure 10: Tensile strength versus polymer weight percent in the sand-polymer composite.

form a solid crust protecting the sand from blowing away by the wind.

The surface crusts, formed from application of polyacrylamide solution, were anchored to the soil below the crust and penetrated easily, making the soil surface resistant to wind erosion. The flat surface held its integrity much better than the hill pattern and protected the sand surface from weathering effects and abrasion. Mechanical properties were used to study the wind erosion resistance and it was found that as the polymer weight percent increased the mechanical properties increased resulting in a stronger composite with high modulus and tensile strength.

It was obvious that there is a continuous and growing demand for sand dune stabilization in the UAE. It is required not only for practicing stable agriculture, but also for road construction and the development of urban and industrial areas. Agricultural land needs to be protected against dune encroachment, which can only be achieved by the application of chemical stabilizers, such as the one prepared in this study and which was successfully tested in the wind tunnel. These stabilizers are environmentally friendly, and they do not produce adverse effects of petroleum mulch. More experiments are required to examine the degradation rate and toxicity of these chemicals subjected to the UAE environment before any attempt to use them on the field.

\section{Conflict of Interests}

The authors declare that there is no conflict of interests regarding the publication of this paper.

\section{Acknowledgment}

The authors are thankful for the help and support that were received from the "Mechanical Engineering and Chemistry Departments" at the UAE University.

\section{References}

[1] A. S. Alsharhan, K. W. Glennie, G. L. Whittle, and C. G. S. Kendall, Quaternary Deserts and Climate Change, A. A. Balkema, Rotterdam, The Netherlands, 1998.

[2] D. J. Gallacher and J. P. Hill, "Effects of camel grazing on the ecology of small perennial plants in the Dubai (UAE) inland desert," Journal of Arid Environments, vol. 66, no. 4, pp. 738750, 2006.

[3] S. M. Lahalih and N. Ahmed, "Effect of new soil stabilizers on the compressive strength of dune sand," Construction and Building Materials, vol. 12, no. 6-7, pp. 321-328, 1998.

[4] Z. Han, T. Wang, Z. Dong, Y. Hu, and Z. Yao, "Chemical stabilization of mobile dunefields along a highway in the Taklimakan Desert of China," Journal of Arid Environments, vol. 68, no. 2, pp. 260-270, 2007.

[5] D. Y. Cheng, X. L. Zhao, and G. D. Kang, Experiment and Research of Shifting sand Stabilization with Asphalt Emulsion. Research of Shifting Sand Control, Ningxia People's Publishing House, Yinchuan, China, 1991.

[6] T. H. Zhang, H. L. Zhao, S. G. Li et al., "A comparison of different measures for stabilizing moving sand dunes in the Horqin Sandy Land of Inner Mongolia, China," Journal of Arid Environments, vol. 58, no. 2, pp. 203-214, 2004.

[7] Z. D. Zhu, X. L. Zhao, and Y. Q. Lin, Sand Control Engineering, China Environmental Science Press, Beijing, China, 2000.

[8] Y. T. Gumaa, I. Haffar, and M. A. Al-Afifi, "Financial appraisal of date-frond mat fence systems for wind erosion control and sand dune stabilization in the arid region of the United Arab Emirates," Journal of Arid Environments, vol. 39, no. 4, pp. 549557,1998

[9] A. M. O. Mohamed, "The role of clay minerals in marly soils on its stability," Engineering Geology, vol. 57, no. 3-4, pp. 193-203, 2000.

[10] H. I. Al-Abdul Wahhab and I. M. Asi, "Improvement of marl and dune sand for highway construction in arid areas," Building and Environment, vol. 32, no. 3, pp. 271-279, 1997. 
[11] S. M. Lahalih, "Development and evaluation of new multipurpose soil additives," Industrial \& Engineering Chemistry Research, vol. 37, no. 2, pp. 420-426, 1998.

[12] M. M. El Banna, "Vulnerability and fate of a coastal sand dune complex, Rosetta-Idku, northwestern Nile Delta, Egypt," Environmental Geology, vol. 54, no. 6, pp. 1291-1299, 2007.

[13] J. W. Vanderhoff, E. B. Bradford, H. L. Tarkowski, J. B. Shaffer, and R. M. Wiley, "Inverse emulsion polymerization," Advances in Chemistry Series, vol. 34, p. 32, 1962.

[14] R. G. Gilbert, Emulsion Polymerization: A Mechanistic Approach, Academic Press, London, UK, 1995.

[15] P. A. Clay and R. G. Gilbert, "Molecular weight distributions in free-radical polymerizations. 1. Model development and implications for data interpretation," Macromolecules, vol. 28, no. 2, pp. 552-569, 1995.

[16] G. H. Alnuaimi, Synthesis and solution characterization of water soluble polyacrylamides and its applications in oil industries [M.S. thesis], UAE University, 2005.

[17] M. A. Mohsin and A. Farah, "Rheological properties of polyacrylamide in enhanced oil recovery applications," in Proceedings of the 2nd Chancellor's Undergraduate Research Award (CURA '08), UAE University, Al-Ain, UAE, May 2008. 

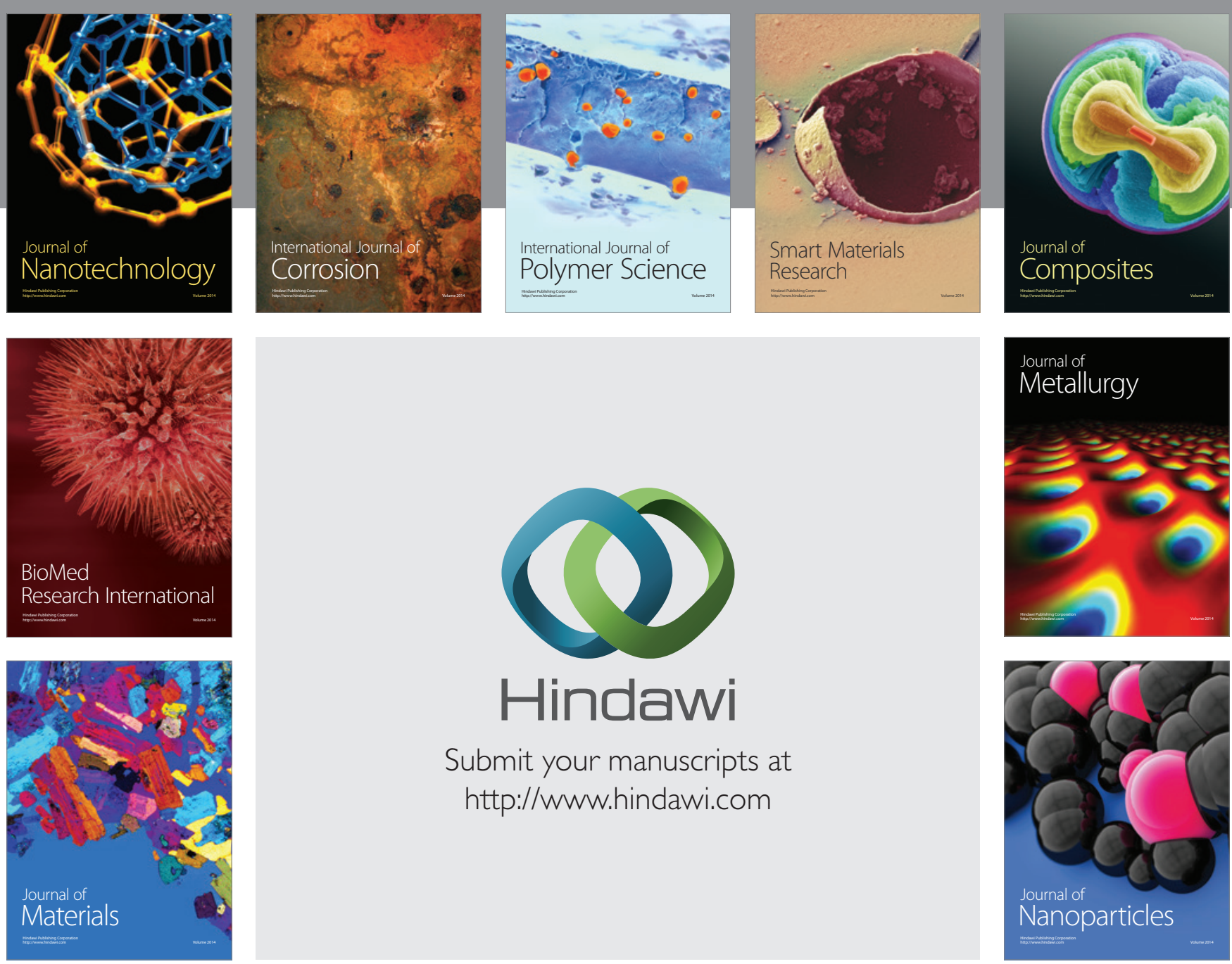

Submit your manuscripts at http://www.hindawi.com
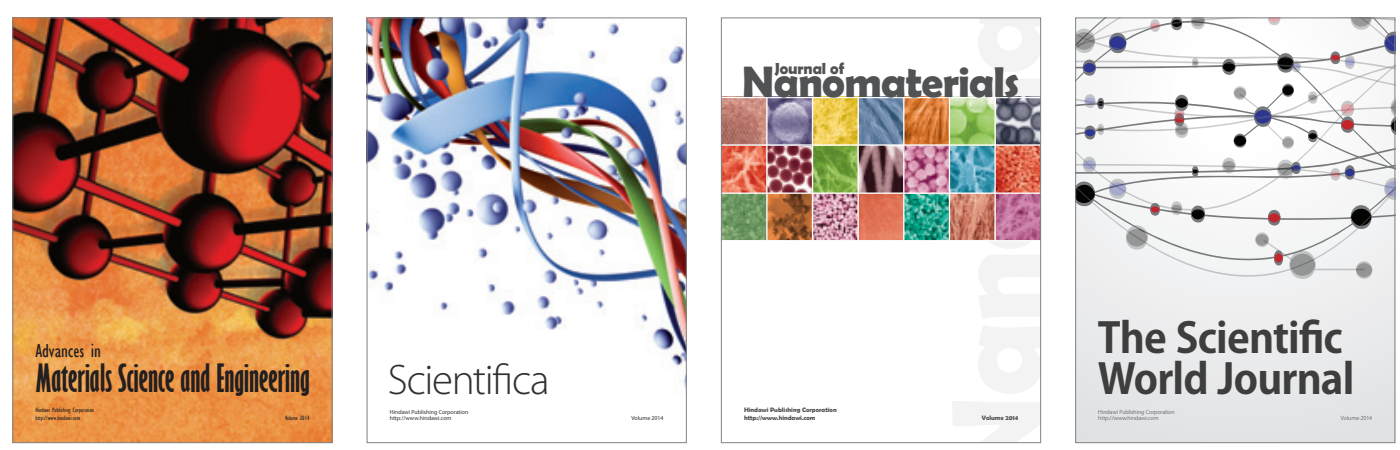

\section{The Scientific World Journal}
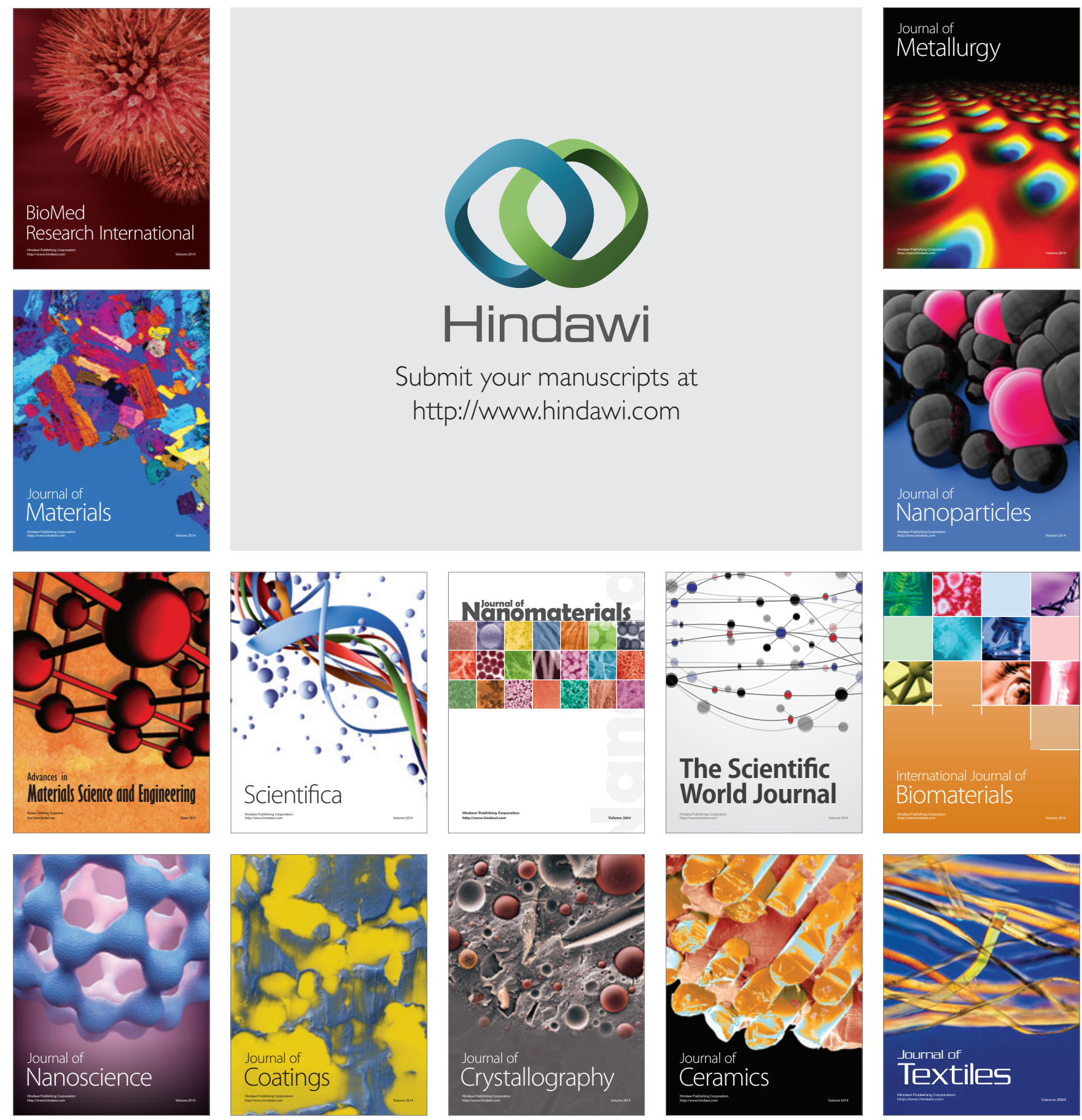